\title{
microRNAs as novel biomarkers of schizophrenia (Review)
}

\author{
JIE WANG $^{1}$, YUHAN WANG ${ }^{1}$, JIANBO YANG ${ }^{2}$ and YUANSHUAI HUANG ${ }^{3}$ \\ ${ }^{1}$ Department of Laboratory Medicine, Luzhou Medical College; Departments of ${ }^{2}$ Nuclear Medicine and ${ }^{3}$ Blood Transfusion, \\ Affiliated Hospital of Luzhou Medical College, Luzhou, Sichuan 646000, P.R. China
}

Received April 8, 2014; Accepted September 11, 2014

DOI: $10.3892 /$ etm.2014.2014

\begin{abstract}
Schizophrenia is a severe mental illness and the most common complex neuropsychiatric disorder. To date, the etiology of schizophrenia is unclear; consequently, the diagnosis of schizophrenia is controversial. Biomarkers that reflect the dysregulations observed in schizophrenia may potentially assist the diagnosis of the disorder. However, the majority of these biomarkers are found in the brain tissue, which is not readily accessible, leading to the search for novel biomarkers that are present in the peripheral blood. microRNAs (miRNAs) are small non-coding RNAs that function as post-transcriptional regulators of gene expression. Previous investigations have revealed that miRNAs may play a potential role in neurodevelopment and maturation, including neurite outgrowth, dendritogenesis and dendritic spine formation. These developments highlight the significance of miRNAs as potential diagnostic biomarkers for schizophrenia. To date, miRNA biomarkers have been predominantly extracted from the brain tissue; however, future studies should examine the use of peripheral blood miRNAs as biomarkers, as these are more accessible.
\end{abstract}

\section{Contents}

1. Introduction

2. miRNAs and schizophrenia

3. miRNAs may be potential biomarkers in the diagnosis of schizophrenia

4. Summary and future prospects

\section{Introduction}

Schizophrenia is a common mental disorder. Schizophrenia is a severe psychiatric disorder that has been found to have

Correspondence to: Dr Yuanshuai Huang, Department of Blood Transfusion, Affiliated Hospital of Luzhou Medical College, 25 Taiping Road, Jiangyang, Luzhou, Sichuan 646000, P.R. China E-mail: hys@live.cn

Key words: schizophrenia, microRNA, etiology, diagnosis, biomarker a lifetime prevalence of $\sim 1 \%$ in a number of population studies (1-3). The condition is characterized by impaired cognition, positive psychotic symptoms, including hallucinations, delusions and disorganized behavior, and negative symptoms, such as social withdrawal and apathy (2). Schizophrenia is a 'complex genetic disease', with an etiology involving multiple genetic and environmental factors. The genetic contribution is significant, since schizophrenia has been shown to have a heritability risk of $\sim 80 \%$, with the risk decreasing by $\sim 50 \%$ for each degree of family relation (3).

microRNAs (miRNA) are the unseen regulators of gene expression. miRNAs are a class of small RNAs found only in eukaryotes, which were first identified less than two decades ago. Modulation of gene expression does not rely solely on regulatory proteins. miRNAs are single-stranded RNA molecules, consisting of 21-23 nucleotides, that are not translated into proteins, but are key to the complex cell machinery responsible for gene expression (4). A previous study estimated that one third of human genes are directly targeted by miRNAs (5). In an attempt to clone the gene responsible for the lin-4 phenotype in roundworms, Lee et al identified miRNAs for the first time as small non-coding RNA molecules (6). To date, 2,652 mature human miRNAs have been identified (www.mirbase.org; accessed 20, June 2013).

The biogenesis of miRNAs is initiated by transcription from intergenic or intron genomic regions into primary-miRNA molecules (pri-miRNAs). Pri-miRNAs are cleaved inside the nucleus by the components of the microprocessor complex, consisting of Drosha and DGCR8, to generate RNA hairpins, known as precursor-miRNA molecules (pre-miRNAs). Pre-miRNA is exported into the cytoplasm and is further cleaved by the RNaseIII enzyme, Dicer; therefore, a duplex of two miRNA strands is formed. Next, the miRNA duplex is unwound and one of the strands is incorporated into a large miRNA-induced silencing complex, which participates in the detection and binding to the 3'-untranslated region of messenger RNAs (mRNAs). As a result, translation is inhibited. However, the underlying mechanisms are yet to be fully understood (Fig. 1) (7).

Genetic basis of miRNA abnormalities in schizophrenia. A hemizygous deletion of a $1.5-3-\mathrm{Mb}$ region of chromosome 22 can lead to the 22q11 deletion syndrome (22q11DS), which is characterized by multiple physical and psychiatric abnormalities. A previous study determined that $\sim 30 \%$ of 


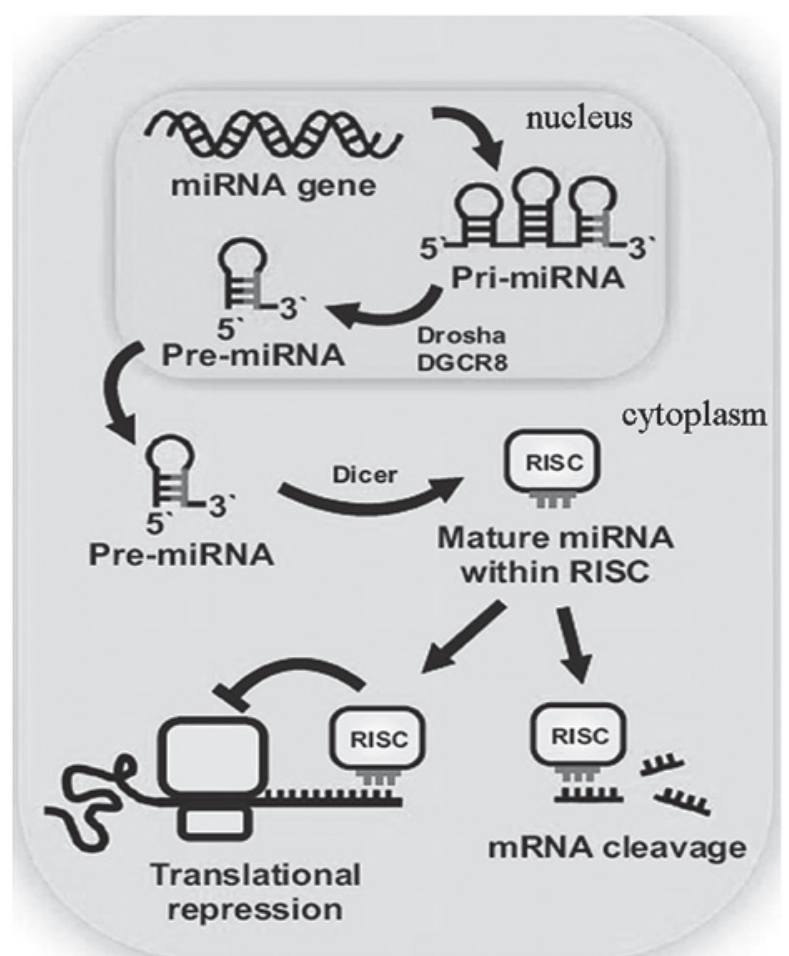

Figure 1. Biogenesis of miRNA [adapted from Bravo and Dinan, 2011 (3)]. Mature miRNA is much smaller compared with the gene involved in encoding the molecule. Pri-miRNA has a hairpin structure with a poly(A) tail and cap. The hairpin molecule is processed by the nuclease, Drosha, and the RNA-binding protein, DGCR8 (known as Pasha in invertebrates), and the resulting molecule is known as pre-miRNA. The small pre-miRNA passes from the nucleus into the cytosol and is processed by the endonuclease, Dicer. Mature miRNA forms part of the RISC and the complex provides the gene silencing capacity of miRNA. miRNA, microRNA; pri-miRNA, primary-miRNA; pre-miRNA, precursor-miRNA; RISC, RNA-induced silencing complex.

22q11DS patients may develop schizophrenia (8). miR-25 and miR-185 are regulators of the sarco/endoplasmic reticulum $\mathrm{Ca}^{2+}$ ATPase (SERCA2), which is responsible for loading $\mathrm{Ca}^{2+}$ into the endoplasmic reticulum. Earls et al found that miR-25 and miR-185 were depleted in mouse models of 22q11DS and restoration of these miRNAs to presynaptic neurons rescued the long-term potentiation of DGCR8 ${ }^{+/-}$mice (1). The authors concluded that miRNA-dependent SERCA2 dysregulation is a pathogenic event in 22q11DS and schizophrenia.

\section{2. miRNAs and schizophrenia}

miRNA function in the nervous system. The intricate architecture of the nervous system and the ability of the neurons for postsynaptic remodeling requires the coordination of complex intracellular networks consisting of molecular signal transduction systems. Due to the abundance of neural networks, gene variants are able to cause system dysfunctions, manifesting as associated neurobehavioral syndromes. Previous studies revealed that post-transcriptional gene regulation by miRNA is an important factor shaping the topography of the neural networks. Over half of miRNAs identified have been shown to be highly or exclusively expressed in the brain, a number of which have been implicated in important aspects of neuronal function (9). miR-124 and miR-9 play a crucial role in neurogenesis; overexpression of these miRNAs decreases the number of astrocytes, whereas inhibition of these miRNAs reduces the number of neurons (10). miR-134 was found to regulate the size of dendritic spines and enrich the synapse dendritic region of rat hippocampal neurons (11). In addition,
miR-134 regulates the translation of Limk1, which is a protein kinase that affects dendritic spine morphology via the regulation of actin filaments (5).

Dysregulation of miRNA in schizophrenia.miRNA has received increasing attention in genetic studies of schizophrenia. A number of studies support the hypothesis that miRNA plays an important role not only in human brain development, but also in brain diseases (12-16). Hunsberger et al hypothesized that miRNA may serve as a unifying link among the structural developmental anomalies, neurotransmitter alterations and response to treatment in schizophrenia (17). The schizophrenia-associated miRNAs are summarized in Table I.

Alterations in the sequence of certain miRNAs leads to the alteration of gene regulation, which contributes to the development of a psychiatric disorder. Perkins et al compared the expression of 264 miRNAs from the prefrontal cortex of patients diagnosed with schizophrenia and 21 individuals not suffering from a psychiatric illness, used as controls (18). Using a custom-made miRNA microarray, the authors identified that the expression of 15 miRNAs decreased and the expression of one miRNA increased in the prefrontal cortex of the schizophrenia patients, when compared with the control individuals.

In addition, Guo et al demonstrated that miR-195 is involved in a complex regulatory network, which affects the signaling pathways considered to be significant in the development of schizophrenia (19).

The gene encoding miR-346 is located in the intron of the glutamate receptor ionotropic $\delta 1$ (GRID1) gene, which is known to be involved in schizophrenia susceptibility (5). Using 
Table I. Schizophrenia-associated miRNA. Adapted from the study by Beveridge and Cairns (36).

\begin{tabular}{|c|c|c|c|}
\hline Study & Type & Brain region & Schizophrenia-associated miRNA \\
\hline Lett et al, 2013 (20) & GWAS & $\begin{array}{l}\text { Hippocampi, } \\
\text { white matter }\end{array}$ & $\operatorname{miR}-137$ \\
\hline Gardiner et al, 2011 (21) & $\begin{array}{l}\text { Peripheral } \\
\text { tissue } \\
\text { (PBMC) }\end{array}$ & N/A & $\begin{array}{l}\text { [Downregulated]miR-107, miR-1275, miR-128, miR-130b }{ }^{*}, \mathrm{miR}-134, \mathrm{miR}-148 \mathrm{~b} \text {, } \\
\text { miR-150*, miR-151-3p, miR-16-2*, miR-181a, miR-200c, miR-224, miR-28-3p, } \\
\text { miR-28-5p, miR-29b-1*, miR-30e, miR-31, miR-329, miR-335, } \\
\text { miR-342-5p,miR-409-3p,miR-431, miR-432, miR-486-3p, miR-487b, miR-544, } \\
\text { miR-574-3p, miR-576-5p, miR-584, miR-625*, miR-664, miR-877, miR-99b }\end{array}$ \\
\hline Lai et al, 2011 (22) & $\begin{array}{l}\text { Peripheral } \\
\text { tissue (PBMC) }\end{array}$ & $\mathrm{N} / \mathrm{A}$ & $\begin{array}{l}\text { [Upregulated] miR-34a, miR-449a, miR-548d, miR-564, miR-572, miR-652 } \\
\text { [Downregulated] miR-432 }\end{array}$ \\
\hline Moreau et al, 2011 (23) & $\begin{array}{l}\text { Postmortem } \\
\text { brain }\end{array}$ & $\begin{array}{l}\text { DLPFC } \\
\text { (BA9) }\end{array}$ & $\begin{array}{l}\text { [Upregulated] miR-148b, miR-151 miR-27b, miR-301, } \operatorname{miR}-545, \text { miR-639 } \\
\text { [Downregulated] miR-106b, miR-138, miR-193b, miR-210, miR-22, } \\
\text { miR-324-3p, miR-338, miR-339, miR-425 }\end{array}$ \\
\hline Santarelli et al, 2011 (24) & $\begin{array}{l}\text { Postmortem } \\
\text { brain }\end{array}$ & $\begin{array}{l}\text { DLPFC } \\
\text { (BA9) }\end{array}$ & $\begin{array}{l}\text { [Upregulated] miR-105, miR-134, miR-148b, miR-150, miR-152, miR-154, } \\
\text { miR-17-5p, miR-187, miR-193a, miR-199a*, miR-199b, miR-222, miR-25, } \\
\text { miR-328, miR-382, miR-409-3p, miR-423, miR-425-5p, miR-433, miR-452, } \\
\text { miR-487a, miR-495, miR-502, miR-512-3p, miR-519c, miR-532, } \\
\text { miR-542-3p, miR-548b, miR-590, miR-592, miR-652, miR-767-5p, miR-92b }\end{array}$ \\
\hline The Schizophrenia & GWAS & N/A & $\operatorname{miR}-137$ \\
\hline
\end{tabular}

Psychiatric GWAS

Consortium, 2011

Beveridge et al, 2010 (25) Postmortem STG

brain (BA22)

[Upregulated] let-7e, miR-107, miR-125b, miR-128a, miR-128b, miR-129, miR-130a, miR-133b, miR-138, miR-146b, miR-148a, miR-150, miR-152, miR-155, miR-15a, miR-15b, miR-16, miR-17-3p, miR-17-5p, miR-181b, miR-195, miR-197, miR-199a*, miR-19a, miR-20a, miR-222, miR-23a， miR-24， miR-26b, miR-26b, miR-27b, miR-28, miR-296, miR-328, miR-330， miR-335， miR-338， miR-339, miR-340, miR-373*, miR-381, miR-409-5p, miR-432*, miR-452*, miR-455, miR-484, miR-485-5p, miR-486, miR-487a, miR-489, miR-494, miR-499, miR-502, miR-517a, miR-517c, miR-518b, miR-519d, miR-520a*, miR-520g, miR-9*, miR-99a

Beveridge et al,2010 (25) Postmortem DLPFC [Upregulated] let-7d, miR-101, miR-105, miR-107, miR-126*, brain (BA9) miR-128a, miR-153, miR-15a, miR-15b, miR-16, miR-16, miR-181a, miR-181b, miR-181b, miR-181d, miR-184, miR-195, miR-199a, miR-20a, miR-219, miR-223, miR-26b, miR-27a, miR-29c, miR-302a*, miR-302b*, miR-31, miR-33, miR-338, miR-409-3p, miR-512-3p, miR-519b, miR-7

$\begin{array}{lllll}\text { Kim et al, } 2010 \text { (26) } & \begin{array}{l}\text { Postmortem } \\ \text { brain }\end{array} & \begin{array}{l}\text { DLPFC } \\ \text { (BA46) }\end{array} & \begin{array}{l}\text { [Upregulated] miR-132, miR-132*, miR-154*, miR-212, miR-34a, } \\ \text { miR-544, miR-7 }\end{array}\end{array}$

$\begin{array}{lll} & \text { brain } & \text { (BA46) } \\ \text { Mellios } \text { et al, 2010 (27) } & \text { Postmortem } & \text { Frontal } \\ & \text { brain } & \text { cortex (BA10) } \\ \text { Xu } \text { et al, 2010 (28) } & \text { miR-SNP } & \text { N/A } \\ \text { Feng } \text { et al, 2009 (29) } & \text { miR-SNP } & \text { N/A }\end{array}$

[Downregulated] miR-30b

miR-24, pre-miR-30e, miR-30e

let-7f-2, miR-188-3p, pre-miR-18b, miR-325-3p, pre-miR-502, pre-miR-505, miR-509-3p, miR-510-3p, miR-660

$\begin{array}{lll}\text { Mellios et al, 2009 (30) } & \begin{array}{l}\text { Postmortem } \\ \text { brain }\end{array} & \begin{array}{l}\text { Frontal } \\ \text { cortex (BA10) }\end{array} \\ \text { Sun } \text { et al, 2009 (31) } & \text { miR-SNP } & \text { N/A } \\ \text { Tabares-Seisdedos } & \text { CNV } & \text { N/A }\end{array}$

[Downregulated] miR-30e, miR-195

miR-502, miR-510

miR-124-1, miR-320, miR-383, miR-486, miR-596, miR-597, miR-598

et al, 2009 (32)

Zhu et al, 2009 (3) Postmortem brain

Beveridge et al, 2008 (33) Postmortem brain

Hansen et al, 2007 (34) miR-SNP N/A

Perkins et al, 2007 (18) Postmortem DLPFC

DLPFC (BA46) miR-346

STG (BA22) miR-181b

miR-198, miR-206

[Upregulated] miR-106b, miR-7

[Downregulated] miR-26b, miR-30b, miR-29b, miR-195, miR-92, miR-30a, miR-30d, miR-20b, miR-29c, miR-29a, miR-212, miR-24, miR-30e, miR-9*

These miRNAs have been shown to exhibit altered expression levels in numerous studies and have exhibited consistent changes in schizophrenia. miRNA, microRNA; GWAS, genome-wide association study; PBMC, peripheral blood mononuclear cells; DLPFC, dorsolateral prefrontal cortex; BA, Brodmann's area; miR-SNP, single nucleotide polymorphism within a microRNA; STG, superior temporal gyrus; CNV, copy-number variant. 
quantitative polymerase chain reaction, Zhu et al detected the expression levels of miR-346 and GRID1 in brain RNA samples of 35 patients with schizophrenia and 34 controls, obtained from the Stanley Medical Research Institute (Chevy Chase, MD, USA) (3). The expression levels of miR-346 and GRID1 were found to be lower in the schizophrenia patients compared with the controls.

In a study of the Chinese population, $\mathrm{Xu}$ et al described a potentially functional variant that affected pre-miR-30e and was closely associated with schizophrenia (28). The variant affected the predicted structure and the release of pre-miRNA, as well as the accuracy of the mature miRNA. Despite the samples being obtained from the peripheral blood, the findings of $\mathrm{Xu}$ et al were comparable to the observations of Perkins et al (18), who detected an increase in the expression level of miR-30e in the prefrontal cortex of patients with schizophrenia.

Smrt et al demonstrated that miR-137 serves as a regulator of adult neural stem cell maturation and migration to the subventricular zone, located around the lateral ventricles, and the subgranular zone of the hippocampus (35). In addition, miR-137 was shown to be highly associated $\left(\mathrm{P}=1.6 \times 10^{-11}\right)$ with schizophrenia in one of the largest genome-wide association studies (36). Since miRNAs are crucial regulators of gene expression, important genetic mechanisms may contribute to the phenotypic heterogeneity. Lett et al made demographics for age-at-onset samples, as well as healthy controls, and their findings indicated that miR-137 plays a considerable role in the variation in phenotypes that is believed to have an important role in clinical outcome and treatment response (20). The authors concluded that the effects of miR-137 on the phenotypic heterogeneity of schizophrenia may occur via neurodevelopmental gene networks. These observations may provide a model for the role of miRNAs in the phenotypic heterogeneity of psychiatric disorders.

\section{3. miRNAs may be potential biomarkers in the diagnosis of schizophrenia}

The diagnosis of schizophrenia is currently based exclusively on signs and symptoms; therefore, a diagnosis requires qualified psychiatric assessment. The majority of studies investigating the genetics of schizophrenia have been limited to protein-coding genes. However, the regulatory role of miRNAs has received increasing attention, with results indicating that miRNAs may contribute to the etiology of schizophrenia. miRNAs are known to influence complex gene networks and pathways, which suggests that they may be potential biomarkers when dysregulated. miRNAs as biomarkers have been shown to be useful in the clinical stratification of neoplasms, and even have a greater prognostic significance compared with mRNAs, which may result from miRNAs being discrete functional entities (36). The expression of peripheral miRNA has also been examined in other neurological disorders. For instance, in Alzheimer's disease, miRNA was found to be altered in peripheral blood mononuclear cells (PBMCs) (37), cerebrospinal fluid and brain tissue (38).

Gardiner et al investigated the expression profile of miRNA in PBMCs of 112 patients with schizophrenia and 76 non-psychiatric controls (21). The authors identified 83 miRNAs that were significantly downregulated in the schizoaffective group, including a large subgroup of miRNAs (20\%) transcribed from a single imprinted locus at the maternally expressed DLK1-DIO3 region on chromosome 14q32. Similarly, Lai et al identified a signature of seven miRNAs in an initial cohort of 30 patients with schizophrenia and 30 controls, which included the upregulated miR-34a, miR-449a, miR-564, miR-548d, miR-572 and miR-652, and downregulated miR-432 (22). The results of the study were subsequently validated in an extended cohort of 60 schizophrenia patients and 30 controls. The expression levels of a number of these miRNAs were found to be correlated with negative symptoms, neurocognitive dysfunction and mismatched negativity performances of the schizoaffective patients. Notably, miR-449a was shown to be closely associated with the majority of features examined in the Wisconsin Card Sorting Test (39), indicating the possible involvement of miR-449a in the executive function of the brain (36).

The majority of previous blood-based gene expression studies on schizophrenia have been limited to the expression of protein-coding genes. A large number of mRNAs have been detected using microarray platforms (40-43), leading to a number of putative mRNAs being associated with schizophrenia; however, the majority of associations have not been possible to replicate in other studies (44). The regulatory role of miRNAs has received increasing attention since miRNAs regulate the expression levels of genes by inhibiting the translation of mRNAs and may be potential biomarkers for schizophrenia (45-49). Since each miRNA can regulate the expression levels of hundreds of target genes, the number of discriminating miRNAs as biomarkers is likely to be much less compared with mRNAs.

Therefore, the aforementioned studies have demonstrated that the peripheral patterns of miRNAs may be used as biomarkers for schizophrenia and are associated with subphenotypes.

\section{Summary and future prospects}

Schizophrenia exhibits a complex neurobehavioral phenotype that is considered to have developed through turbulences in the neural circuitry and synaptic function. Due to the abundance of neural networks, various combinations of gene variants can cause system dysfunctions, manifesting as associated neurobehavioral syndromes. miRNAs appear to be important in neural networks and are highly expressed in the brain, emerging as key regulators of numerous neurodevelopmental and neurological processes. Dysregulation of miRNAs leads to pervasive changes and defects of the nervous system, which may assist investigations into the pathophysiology and neuropathology of schizophrenia. Due to their tuning effect on numerous proteins, miRNA biomarkers for schizophrenia and associated phenotypes may ultimately provide the basis for early detection, disease stratification and prediction of response to drugs and side-effects; however, mood swings can affect the expression of certain miRNAs (50), and this may be a disadvantage of using miRNAs to diagnose schizophrenia.

miRNAs have the following advantages: i) miRNA remains stable despite changes in temperature, $\mathrm{pH}$ or physical state; 
ii) the expression of miRNAs is specific with no difference in gender or in individual; iii) miRNAs in serum and plasma can be quantified in vitro (51); thus, miRNAs are promising biomarkers instead of proteins. To date, miRNAs extracted from brain tissue, cerebrospinal fluid and PBMCs have been used as biomarkers in the diagnosis of schizophrenia; furthermore, the decoding of the miRNA genome may contribute to elucidating the etiology and improving the treatment of schizophrenia. miRNAs extracted from other peripheral sources, however, have not been investigated. Future studies should investigate miRNAs from other peripheral fluids, including saliva and urine, as these may also be potential biomarkers in the diagnosis of schizophrenia.

\section{References}

1. Earls LR, Fricke RG, Yu J, Berry RB, Baldwin LT and Zakharenko SS: Age-dependent microRNA control of synaptic plasticity in 22q11 deletion syndrome and schizophrenia. J Neurosci 32: 14132-14144, 2012.

2. Abdolmaleky HM and Thiagalingam S: Can the schizophrenia epigenome provide clues for the molecular basis of pathogenesis? Epigenomics 3: 679-683, 2011.

3. Zhu Y, Kalbfleisch T, Brennan MD and Li Y: A microRNA gene is hosted in an intron of a schizophrenia-susceptibility gene. Schizophr Res 109: 86-89, 2009.

4. Guarnieri DJ and DiLeone RJ: microRNAs: a new class of gene regulators. Ann Med 40: 197-208, 2008.

5. Bravo JA and Dinan TG: microRNAs: a novel therapeutic target for schizophrenia. Curr Pharm Des 17: 176-188, 2011.

6. Lee RC, Feinbaum RL and Ambros V: The C. elegans heterochronic gene lin-4 encodes small RNAs with antisense complementarity to lin-14. Cell 75: 843-854, 1993.

7. Bartel DP: microRNAs: genomics, biogenesis, mechanism, and function. Cell 116: 281-297, 2004.

8. Williams NM: Molecular mechanisms in 22q11 deletion syndrome. Schizophr Bull 37: 882-889, 2011.

9. Cao X, Yeo G, Muotri AR, Kuwabara T and Gage FH: Noncoding RNAs in the mammalian central nervous system. Annu Rev Neurosci 29: 77-103, 2006.

10. Vo N, Klein ME, Varlamova O, et al: A cAMP-response element binding protein-induced microRNA regulates neuronal morphogenesis. Proc Natl Acad Sci USA 102: 16426-16431, 2005.

11. Schratt GM, Tuebing F, Nigh EA, et al: A brain-specific microRNA regulates dendritic spine development. Nature 439: 283-289, 2006

12. Sepramaniam S, Tan JR, Tan KS, et al: Circulating microRNAs as biomarkers of acute stroke. Int J Mol Sci 15: 1418-1432, 2014.

13. Ma L, Wei L, Wu F, et al: Advances with microRNAs in Parkinson's disease research. Drug Des Devel Ther 7: 1103-1013, 2013.

14. Müller M, Kuiperij HB, Claassen JA, et al: MicroRNAs in Alzheimer's disease: differential expression in hippocampus and cell-free cerebrospinal fluid. Neurobiol Aging 35 :152-158, 2014.

15. Kumar P, Dezso Z, MacKenzie C, et al: Circulating miRNA biomarkers for Alzheimer's disease. PLoS One 8: e69807, 2013.

16. Shi W, Du J, Qi Y, et al: Aberrant expression of serum miRNAs in schizophrenia. J Psychiatr Res 46: 198-204, 2012.

17. Hunsberger JG, Austin DR, Chen G and Manji HK: microRNAs in mental health: from biological underpinnings to potential therapies. Neuromolecular Med 11: 173-182, 2009.

18. Perkins DO, Jeffries CD, Jarskog LF, et al: microRNA expression in the prefrontal cortex of individuals with schizophrenia and schizoaffective disorder. Genome Biol 8: R27, 2007.

19. Guo AY, Sun J, Jia P and Zhao Z: A novel microRNA and transcription factor mediated regulatory network in schizophrenia. BMC Syst Biol 4: 10, 2010.

20. Lett TA, Chakavarty MM, Felsky D, et al: The genome-wide supported microRNA-137 variant predicts phenotypic heterogeneity within schizophrenia. Mol Psychiatry 18: 443-450, 2013

21. Gardiner E, Beveridge NJ, Wu JQ, et al: Imprinted DLK1-DIO3 region of $14 \mathrm{q} 32$ defines a schizophrenia-associated miRNA signature in peripheral blood mononuclear cells. Mol Psychiatry 17: 827-840, 2012.
22. Lai CY, Yu SL, Hsieh MH, et al: microRNA expression aberration as potential peripheral blood biomarkers for schizophrenia. PLoS One 6: e21635, 2011.

23. Moreau MP, Bruce SE, David-Rus R, et al: Altered microRNA expression profiles in postmortem brain samples from individuals with schizophrenia and bipolar disorder. Biol Psychiatry 69: 188-193, 2011.

24. Santarelli DM, Beveridge NJ, Tooney PA and Cairns MJ: Upregulation of dicer and microRNA expression in the dorsolateral prefrontal cortex Brodmann area 46 in schizophrenia. Biol Psychiatry 69: 180-187, 2011.

25. Beveridge NJ, Gardiner E, Carroll AP, et al: Schizophrenia is associated with an increase in cortical microRNA biogenesis. Mol Psychiatry 15: 1176-1189, 2010.

26. Kim AH, Reimers M, Maher B, et al: MicroRNA expression profiling in the prefrontal cortex of individuals affected with schizophrenia and bipolar disorders. Schizophr Res 124: 183-191, 2010.

27. Mellios N, Galdzicka M, Ginns E, et al: Gender-specific reduction of estrogen-sensitive small RNA, miR-30b, in subjects with schizophrenia. Schizophr Bull 38: 433-443, 2010.

28. Xu Y, Li F, Zhang B, et al: microRNAs and target site screening reveals a pre-microRNA-30e variant associated with schizophrenia. Schizophr Res 119: 219-227, 2010.

29. Feng J, Sun G, Yan J, et al: Evidence for X-chromosomal schizophrenia associated with microRNA alterations. PLoS One 4: e6121, 2009.

30. Mellios N, Huang HS, Baker SP, et al: Molecular determinants of dysregulated GABAergic gene expression in the prefrontal cortex of subjects with schizophrenia. Biol Psychiatry 65: 1006-1014, 2009

31. Sun G, Yan J, Noltner K, et al: SNPs in human miRNA genes affect biogenesis and function. RNA 15: 1640-1651, 2009.

32. Tabarés-Seisdedos R and Rubenstein JL: Chromosome 8p as a potential hub for developmental neuropsychiatric disorders: implications for schizophrenia, autism and cancer. Mol Psychiatry 14: 563-589, 2009.

33. Beveridge NJ, Tooney Pa, Carroll AP, et al: Dysregulation of miRNA $181 \mathrm{~b}$ in the temporal cortex in schizophrenia. Hum Mol Genet 17: 1156-1168, 2008.

34. Hansen T, Olsen L, Lindow M, et al: Brain expressed microRNAs implicated in schizophrenia etiology. PLoS One 2: e873, 2007.

35. Smrt RD, Szulwach KE, Pfeiffer RL, et al: microRNA miR-137 regulates neuronal maturation by targeting ubiquitin ligase mind bomb-1. Stem Cells 28: 1060-1070, 2010.

36. Beveridge NJ and Cairns MJ: microRNA dysregulation in schizophrenia. Neurobiol Dis 46: 263-271, 2012.

37. Schipper HM, Maes OC, Chertkow HM and Wang E: microRNA expression in Alzheimer blood mononuclear cells. Gene Regul Syst Bio 1: 263-274, 2007.

38. Cogswell JP, Ward J, Taylor IA, et al: Identification of miRNA changes in Alzheimer's disease brain and CSF yields putative biomarkers and insights into disease pathways. J Alzheimers Dis 14: 27-41, 2008 .

39. Berg EA: A simple objective technique for measuring flexibility in thinking. J Gen Psychol 39: 15-22, 1948.

40. Vawter MP, Ferran E, Galke B, Cooper K, Bunney WE and Byerley W: microarray screening of lymphocyte gene expression differences in a multiplex schizophrenia pedigree. Schizophr Res 67: 41-52, 2004.

41. Glatt SJ, Everall IP, Kremen WS, et al: Comparative gene expression analysis of blood and brain provides concurrent validation of SELENBP1 up-regulation in schizophrenia. Proc Natl Acad Sci USA 102: 15533-15538, 2005.

42. Sullivan PF, Fan C and Perou CM: Evaluating the comparability of gene expression in blood and brain. Am J Med Genet B Neuropsychiatr Genet 141B: 261-268, 2006.

43. Kuzman MR, Medved V, Terzic J and Krainc D: Genome-wide expression analysis of peripheral blood identifies candidate biomarkers for schizophrenia. J Psychiatr Res 43: 1073-1077, 2009.

44. Yao Y, Schröder J and Karlsson H: Verification of proposed peripheral biomarkers in mononuclear cells of individuals with schizophrenia. J Psychiatr Res 42: 639-643, 2008.

45. Perkins DO, Jeffries C and Sullivan P: Expanding the "central dogma': the regulatory role of nonprotein coding genes and implications for the genetic liability to schizophrenia. Mol Psychiatry 10: 69-78, 2005.

46. Cheng HY, Papp JW, Varlamova O, et al: microRNA modulation of circadian-clock period and entrainment. Neuron 54: 813-829, 2007. 
47. Kocerha J, Faghihi MA, Lopez-Toledano MA, et al: microRNA-219 modulates NMDA receptor-mediated neurobehavioral dysfunction. Proc Natl Acad Sci USA 106: 3507-3512, 2009.

48. Coyle JT: microRNAs suggest a new mechanism for altered brain gene expression in schizophrenia. Proc Natl Acad Sci USA 106: 2975-2976, 2009.

49. Miller BH and Wahlestedt C: microRNA dysregulation in psychiatric disease. Brain Res 1338: 89-99, 2010.
50. Zhou R, Yuan P, Wang Y, et al: Evidence for selective microRNAs and their effectors as common long-term targets for the actions of mood stabilizers. Neuropsychopharmacology 34: 1395-1405, 2009.

51. Mitchell PS, Parkin RK, Kroh EM, Fritz BR, Wyman SK, Pogosova-Agadjanyan EL, et al: Circulating microRNAs as stable blood-based markers for cancer detection. Proc Natl Acad Sci USA 105: 10513-10518, 2008. 